\title{
Characterization and recovery of mercury from spent fluorescent lamps
}

\author{
Min Jang, Seung Mo Hong, Jae K. Park
}

Introduction

The presence of mercury in the environment is a persistent and increasing problem. Since fluorescent lamps, which rely on mercury for their operation, are more energy-efficient than incandescent lamps (Hildenbrand et al., 2000; Thaler et al., 1995), their extensive use over the years has caused growing concerns over their proper disposal. The National Electrical Manufactures Association (NEMA) has projected that approximately 680 million fluorescent lamps will be disposed of in the United States in 2004 (NEMA, 2000). Although the total amount of mercury in fluorescent lamps varied significantly depending on the type of lamp and year of manufacture, the amount of mercury in fluorescent lamps has decreased over time due to the efforts to decrease environmental contamination. The mercury content of spent fluorescent lamps has been reported to be between 0.72 and $115 \mathrm{mg} / \mathrm{lamp}$ with an average mercury content of about $30 \mathrm{mg} / \mathrm{lamp}$ in 1994 (Battye et al., 1994; Truesdale et al., 1993) NEMA reported that the average mercury content of a 4-foot lamp was $11.6 \mathrm{mg}$ in 1999.

However, it has been found that many fluorescent lamps contain sufficient quantities of mercury to fail the toxicity characteristics for mercury when they are disposed.

Due to persistent mismanagement of lamps, the Environmental 
Protection Agency (EPA) recently published the new hazardous waste lamp rule that adds waste lamps to the federal list of universal wastes, which are regulated under the Resource Conservation and Recovery Act (RCRA) (EPA, 1999). Furthermore, land disposal restriction (LDR) treatment requirements for characteristic wastes went into effect on May 8, 1992. Thus, before hazardous wastes containing mercury can be landfilled, they must be treated to below the LDR standards. The LDR standard specified in 40 CFR 268.40 for lamps that are mercury characteristic hazardous wastes is $0.025 \mathrm{mg} / \mathrm{L}$, as determined by the TCLP test (Walter, 2001). One way to avoid the treatment of fluorescent lamps to LDR standards is to recycle the lamps.

Most lamp recyclers in the United States employ the dry recycling process which generates four products:

mercury-contaminated phosphor powder, mercurycontaminated filters, crushed glass, and aluminum end caps. The entire system is operated under negative pressure to minimize mercury emissions to the atmosphere (Tanel et al., 1998). The spent fluorescent lamps are first broken in a crushing unit. During crushing, a vacuum system placed under crusher grates collects the air and crushed materials, preventing the mercury from escaping through the feed tube. The materials are passed through a cyclone separator, in which aluminum end caps and broken glass are separated and phosphor powder containing mercury is transferred to an enclosed auger conveyer. The elemental mercury released in the vapor phase is captured by carbon filters, which are ultimately sent out for mercury reclamation. Mercury-contaminated phosphor powder and filters containing elemental mercury are shipped as hazardous waste to a mercury refining company for retorting to recover elemental mercury and phosphor powder. These mercury-containing wastes are placed in a retort and heated above the boiling point 
of mercury (375_C) for 4-20 h. The vaporized mercury is condensed in the scrubber and then collected in a decanter.

Additional treatments such as nitric acid bubbling may be required to remove impurities from the mercury before it can be reused. The crushed glass is usually sent for reuse in other applications such as the manufacture of fiberglass insulation, road material or a multitude of other products. Aluminum end caps are sold to aluminum manufacturers to smelt into aluminum sheet or other products for resale as raw material.

Although the entire system of dry recycling procedure can minimize mercury emissions to the atmosphere, the effect of mercury adsorbed onto the glass on its recyclability and public health has not been investigated to date. There are also few studies on how much mercury is partitioned to the different compartments of spent and new fluorescent lamps.

Accordingly, in this study, wet chemical analysis (WCA) methods were utilized to achieve the following objectives: (1) determine partitioning of mercury among the vapor phase, loose phosphor produced during breaking and washing steps, lamp glass matrix, phosphor powder attached on the glass, and aluminum end caps for different types of spent and new fluorescent lamps; (2) compare mercury concentrations in glasses taken from lamp recyclers with the results of simulated laboratory recycling tests; (3) evaluate the effectiveness of mercury recovery methods from lamp components, such as acid solutions and heating process for extracting mercury from spent fluorescent lamps.

Materials and methods

Different types of spent fluorescent lamps were obtained from the University of Wisconsin-Madison Physical Plant. New lamps, manufactured by several companies, were purchased in retail stores in Madison, Wisconsin. Recycled glass samples were obtained from 
two commercial fluorescent lamp recyclers located in Wisconsin for TCLP tests and the amount of the total mercury in the glass samples was measured. The spent and new fluorescent lamps were used to determine partitioning of mercury in the following phases: (1) vaporized elemental mercury; (2) mercury released from phosphor powders, which were physically detached during lamp breaking and washing with deionized water; (3) mercury in the phosphor powder attached in the glass;

(4) mercury adsorbed into the glass matrices; and (5) mercury adsorbed onto the end caps.

Specifications of spent and new fluorescent lamps tested in the study are summarized in Table 1. Two different types of lamps were tested: T8 and T12. T8 lamps are $26 \mathrm{~mm}$ in diameter and range from 600 to $1800 \mathrm{~mm}$ in length while T12 lamps are $38 \mathrm{~mm}$ in diameter and can range from 600 to $2400 \mathrm{~mm}$ in length. The T8 linear fluorescent lamp is the modern and energy efficient alternative to the T12 lamp. In this study, different types of spent T8, spent T12, and new T12 were selected with the same length of $1800 \mathrm{~mm}$. Three different types of spent T8 were tested. New and spent T12 lamps manufactured by two companies were selected because their consumption is larger than other products in Wisconsin.

All mercury analyses were based on Method 7471B (Mercury in Solid or Semisolid Waste - Manual Cold Vapor Technique) from EPA_s "Test Methods for Evaluating Solid Waste - Physical/Chemical Method," (SW-

846) (EPA, 1998). In this study, it was not possible to obtain reproducible results from total mercury analysis because Method 7471B specifies a sample size of $0.6 \mathrm{~g}$ which is too small to represent the total sample. There-fore, several modifications to Method 7471B were made for total mercury analysis so as to obtain reliable results for the entire weight of the lamp. The mercury analysis of each component of fluorescent lamps is described below. 
2.1. Sample preparation and partitioning of mercury

eionized water. After weighing, each lamp was placed

onto a $140 \cdot 25 \mathrm{~cm}$ laboratory bench paper. A dip

scratch was made with a glass-cutter on the center of the fluorescent lamp. The elemental mercury in the vapor phase was captured by a collection system that was composed of a wide-mouth, cone shaped funnel (105 mm ID) connected to a peristaltic pump with a teflon tube. The teflon tube was immersed into $100-\mathrm{mL}$ of mixed acid solution. The mixed acid solution was prepared with nitric acid $5 \%(\mathrm{v} / \mathrm{v})$ and hydrochloric acid $5 \%(\mathrm{v} / \mathrm{v})$ following the recommendation of Dominski (1985). Before crushing the lamp, the peristaltic pump was set on maximum speed. The lamp was broken into two parts with the stainless steel bar directly under the collection funnel. After collecting the mercury in the vapor phase into the mixed acid solution, the solution was analyzed for mercury immediately with the manual cold vapor method. The broken lamp was then separated into its component parts for testing. First of all, the following experiments were conducted to investigate how much mercury can be released from the loose phosphor powder produced through breaking the lamp and washing the inside of fluorescent lamps with deionized water.

The inside of the lamp was washed with about $50 \mathrm{~mL}$ of deionized water for $30 \mathrm{~min}$. The solution was then collected in a $100-\mathrm{mL}$ volumetric flask. The mixed acid solution of hydrochloric acid and nitric acid was added into this solution to be $5 \%(\mathrm{v} / \mathrm{v})$ for each acid solution. Next, the total volume was adjusted to $100 \mathrm{~mL}$ with additional deionized water. The solution was stirred at room temperature for $24 \mathrm{~h}$ before analysis by the manual cold vapor method. The lamp glasses were dried under vacuum at room temperature for $4 \mathrm{~h}$ without collection of mercury and wrapped with laboratory bench paper and then shattered into $2-3$ in. pieces with a hammer. The glass pieces were inserted into the grinder 
and the lid was closed tightly. The glass was gently

pulverized into small particles for $10 \mathrm{~min}$. in order to obtain more homogeneous samples for mercury analyses.

The pulverized particles were collected in a pre-cleaned $300-\mathrm{mL}$ capped vessel. Before analysis, the samples were preserved in a refrigerator at 4 _C. The pulverized glass samples were used for several mercury analysis including TCLP and mercury extraction tests.

The separated aluminum end caps free of phosphor powder from each lamp were weighed and analyzed for mercury using method 7471B. Both end caps were added to a pre-cleaned $300-\mathrm{mL}$ capped-vessel and digested with a mixture of $25 \mathrm{~mL}$ of reagent water, $25 \mathrm{~mL}$ of aqua regia, and $12.5 \mathrm{~mL}$ of potassium permanganate solution, which can be designated as the digestion mixture. Aqua regia was prepared immediately before use by carefully adding three volumes of concentrated $\mathrm{HCl}$ to one volume of concentrated $\mathrm{HNO}_{3}$. Potassium permanganate was prepared at $5 \%$ based on the weight to volume basis. The capped vessel was mixed using a shaker for $18 \pm 2 \mathrm{~h}$ at room temperature. The supernatant was then filtered with a $0.45-1 \mathrm{~m}$ pore size filter and diluted with mixed acid solution of hydrochloric acid $(5 \%, v / v)$ and nitric acid $(5 \%, v / v)$ to stay within the range of the standard curve.

To analyze the total mercury concentration of the lamp glasses, approximately $50 \mathrm{~g}$ of the pulverized glass from each lamp tested in this study and lamp glasses obtained from the recyclers was weighed and placed in a $300-\mathrm{mL}$ capped vessel. The mercury extraction and analysis methods were the same as those of end-caps described above.

Since total mercury concentration of lamp glasses includes both mercury immobilized into phosphor powder attached on the glass, and mercury partitioned to the glass matrices, the following experiments were performed to determine how much mercury was partitioned 
to the glass matrices. Only spent T12 fluorescent lamps were used for this experiment. About $20 \mathrm{~g}$ of the pulverized glass was placed into a $500-\mathrm{mL}$ volumetric flask. Then, $400 \mathrm{~mL}$ of deionized water was poured over the glass sample. The flask was mixed vigorously using a shaker for $18 \pm 2 \mathrm{~h}$ at room temperature. The supernatant including the phosphor powder was discarded, and this procedure was repeated several times to remove all phosphor powder until the glass samples became transparent. Since mercury concentrations in the entire lamp and washed glass were measured, the mercury concentration in the supernatent was not measured. In order to measure the mercury concentrations of glasses without phosphor powders, these samples were dried at room temperature for $24 \mathrm{~h}$. Raposo et al. (2003) showed that mercury extinction started above 250 _C in the TD (thermal desorption) profile of mercury within a glass sample of a spent fluorescent lamp. This might be caused by a strong linkage of mercury into the matrix of glass. Therefore, the mercury could not be released during the drying step at room temperature for $24 \mathrm{~h}$. Samples were weighed again and placed in a $300-\mathrm{mL}$ capped vessel. As described previously, these samples were digested with the digestion mixture. The mercury extraction procedure and analysis were the same as the previous experimental procedure of mercury analysis for glass.

Full text is available at : 